\title{
Preparation of an exciton condensate of photons on a 53-qubit quantum computer
}

\author{
LeeAnn M. Sager, Scott E. Smart, and David A. Mazziotti $\odot^{*}$ \\ Department of Chemistry and James Franck Institute, University of Chicago, Chicago, Illinois 60637, USA
}

(Received 11 May 2020; accepted 25 September 2020; published 9 November 2020)

\begin{abstract}
Quantum computation promises an exponential speedup of certain classes of classical calculations through the preparation and manipulation of entangled quantum states. So far, most molecular simulations on quantum computers, however, have been limited to small numbers of particles. Here we prepare a highly entangled state on a 53-qubit IBM quantum computer, representing 53 particles, which reveals the formation of an exciton condensate of photon particles and holes. While the experimental realization of ground state exciton condensates remained elusive for more than 50 years, such condensates were recently achieved for electron-hole pairs in graphene bilayers and metal chalcogenides. Our creation of ground state photon condensates has the potential to further the exploration of exciton condensates, and this novel preparation may play a role in realizing efficient room-temperature energy transport.
\end{abstract}

DOI: 10.1103/PhysRevResearch.2.043205

\section{INTRODUCTION}

Exciton condensation is defined by the condensation of particle-hole pairs (excitons) into a single quantum state to create a superfluid. The superfluidity of electron-hole pairs-while, by definition, incapable of involving either the frictionless flow of matter or charge-does involve the nondissipative transfer of energy [1,2]. As such, understanding and exploiting the superfluid properties of exciton condensates may be instrumental in the effort to design wires and electronic devices with minimal loss of energy. Consequently, considerable theoretical and experimental investigation has centered on exciton condensation in recent years [1,3-9].

While excitons form spontaneously in semiconductors and insulators and while the binding energy of the excitons can greatly exceed their thermal energy at room temperature, they recombine too quickly to allow for formation of a condensate in a simple manner. To combat recombination, the coupling of excitons to polaritons-which requires the continuous input of light $[9,10]$ - and the physical separation of electrons and holes into bilayers - which involves impractically high magnetic fields and/or low temperatures [3,4,9,11,12]—are employed. Thus, a new, more practical avenue for the creation of exciton condensates and the study of their properties is desired.

Computation on quantum devices has recently been employed to explore strongly correlated quantum matter [13], as superconducting circuits allow for precise manipulation of the strongly interacting qubits to create specified quantum states

\footnotetext{
*Corresponding author: damazz@uchicago.edu

Published by the American Physical Society under the terms of the Creative Commons Attribution 4.0 International license. Further distribution of this work must maintain attribution to the author(s) and the published article's title, journal citation, and DOI.
}

populated by microwave photons [13-18]. Here we prepare and measure an exciton condensate of photons on a quantum computer. Quantum computation should be particularly suited to the exploration of exciton condensation as the binary nature of an individual qubit can be interpreted as a site consisting of one fermion and two orbitals; extrapolating, a system of $N$ qubits can be viewed as $N$ degenerate sites each consisting of one fermion and two distinct orbitals. (See Fig. 1 for an example of how the $|010\rangle$ qubit state is interpreted in this paradigm.) We use such a Hamiltonian of $N$ fermions in two $N$-degenerate levels, known as the Lipkin Hamiltonian [19-23] (see the Supplemental Material [43] for details), to prepare a molecular-scale exciton condensate by tuning its interaction parameter to the large-coupling limit. Because each transmon qubit on the quantum computer utilized for this study can be interpreted as an anharmonic oscillator [13-18] whose natural particles are photons (see Ref. [13]), the exciton condensates we construct can be viewed as being composed of photon-hole pairs condensing into single quantum statesi.e., exciton condensates of photons on a quantum computer.

We use the theoretical signature of exciton condensationderived by Rosina and Garrod [5,24] — to probe the extent of exciton condensation for a wide range of preparations through simulation and physical quantum computation experiments. From analysis of the natural occupation numbers of these preparations, we establish that prepared states with orbital occupation numbers consistent with the Greenberger-HorneZeilinger (GHZ) state-including but not limited to the GHZ state-demonstrate maximal exciton condensation for three qubits. Further, we establish through simulation that for any number of qubits, the GHZ state exhibits maximal character of exciton condensation, demonstrating that the "maximal entanglement" of the GHZ state-for all $N$-corresponds to the entanglement of particle-hole pairs. Through preparing and probing the GHZ states on quantum devices, character of exciton condensation is experimentally observed in systems composed of up to 53 qubits, although decoherence in 
the higher-qubit systems leads to multiple eigenstates of the particle-hole reduced density matrix $\left[{ }^{2} \tilde{G}\right.$; Eq. (3)] demonstrating excitonic character instead of a single, maximally entangled eigenstate. Specifically, as the GHZ state is prepared here on transmon qubits, the realization of exciton condensation on the experimental quantum devices of 3 to 53 qubits can be interpreted as the entanglement of photon-hole pairs, i.e., the experimental observation of exciton condensates of photons for systems of 3 to 53 qubits.

\section{SIGNATURE OF EXCITON CONDENSATION}

Condensation phenomena have been an active area of research since 1924 when Einstein and Bose first introduced their ideal "Bose-Einstein" gas [25,26]. The identical particles composing this gas-bosons-are able to aggregate into a single quantum ground state when sufficiently cooled [26], which leads to superfluidity of the constituent bosons [27,28]. In 1940, Pauli established the relationship between spin and statistics, demonstrating that particles with integral spin values (bosons) obey Bose-Einstein statistics and hence may form a condensate [29]. Particles with half-integer spins (fermions), in contrast, must obey the Pauli exclusion principle and are therefore unable to condense into a single quantum state to form a condensate. However, pairs of fermionsforming an overall bosonic state-can condense. In a system of fermionic particles, this pairing can be accomplished through either particle-particle or particle-hole pairing. The condensation of particle-particle pairs into a single quantum state is termed fermion-pair condensation with the resultant superfluidity of fermion pairs causing superconductivity [30]; likewise, the condensation of particle-hole pairs (excitons) into a single quantum state is termed exciton condensation with the resultant superfluidity of exciton pairs causing the nondissipative transfer of energy [1].

In order to computationally probe the presence and extent of condensation behavior, it is useful to establish a calculable, characteristic property. As proven independently by Yang and Sasaki [31,32], the quantum signature of fermion condensation is associated with a large eigenvalue of the particle-particle reduced density matrix (RDM) with elements given by

$$
{ }^{2} D_{k, l}^{i, j}=\left\langle\Psi\left|\hat{a}_{i}^{\dagger} \hat{a}_{j}^{\dagger} \hat{a}_{l} \hat{a}_{k}\right| \Psi\right\rangle,
$$

where $|\Psi\rangle$ is an $N$-fermion wave function, the Roman indices correspond to one-fermion orbitals in a finite basis set with rank $r$, and $\hat{a}^{\dagger}$ and $\hat{a}$ are fermionic creation and annihilation operators, respectively. We denote the largest eigenvalue of the particle-particle RDM as $\lambda_{D}$ and use this value as a signature of the extent of fermion pair condensation, with values above 1 demonstrating condensation.

In analogy to the signature of fermion pair (particleparticle) condensation being a large eigenvalue of the particle-particle RDM, one may assume the quantum signature of exciton (particle-hole) condensation to be a large eigenvalue in the particle-hole RDM [5,24,33] with elements given by

$$
{ }^{2} G_{k, l}^{i, j}=\left\langle\Psi\left|\hat{a}_{i}^{\dagger} \hat{a}_{j} \hat{a}_{l}^{\dagger} \hat{a}_{k}\right| \Psi\right\rangle
$$

However, there exist two large eigenvalues for the particlehole RDM, one of which corresponds to a ground-state-toground-state transition (not exciton condensation). In order to eliminate this extraneous large eigenvalue, the modified particle-hole matrix with the ground state resolution removed,

$$
{ }^{2} \tilde{G}_{k, l}^{i, j}={ }^{2} G_{k, l}^{i, j}-{ }^{1} D_{k}^{i 1} D_{l}^{j},
$$

is constructed. Garrod and Rosina [24] have shown that-for an $N$-fermion system-the eigenvalues of the ${ }^{2} \tilde{G}$ matrix are zero or 1 in the noninteracting limit and bounded above by $\frac{N}{2}$ in the limit of strong correlation. We denote the largest eigenvalue of the modified particle-hole RDM as $\lambda_{G}$ and use this value as a signature of the presence and extent of exciton condensation.

Note that while we use fermionic creation and annihilation operators to be consistent with the traditional approach to condensation in which particle-particle pairing occurs when two electrons form a Cooper pair and particle-hole pairing occurs when an electron and an electron-hole become paired, bosonic operators can equivalently be employed. A large eigenvalue in the boson-boson reduced density matrix or a large eigenvalue in the modified boson-hole reduced density matrix would equivalently indicate boson-boson pairs or boson-hole pairs condensing into a single quantum state and hence demonstrating condensation, respectively.

\section{RESULTS}

\section{A. Condensation with 3 excitons}

Three-qubit systems-which correspond to three fermions in six orbitals [34] —are the smallest systems to possess nontrivial classes of entanglement. Hence, in this study, these minimally small, three-qubit systems are first thoroughly explored in order to obtain insights on the preparation and characteristics of exciton condensates that are later employed to guide the investigation of larger-qubit systems.

To this end, the three-qubit preparation

$$
\begin{aligned}
|\Psi\rangle & =C_{3}^{2} R_{y, 3}\left(\theta_{3}\right) C_{1}^{2} R_{y, 1}\left(\theta_{2}\right) C_{1}^{3} R_{y, 1}\left(\theta_{1}\right)|000\rangle \\
& =\alpha|000\rangle+\beta|011\rangle+\gamma|101\rangle+\delta|110\rangle,
\end{aligned}
$$

which —as shown in Ref. [34]—is a minimalistic three-qubit preparation known to effectively span all real, 1-qubit occupations, is utilized to systematically prepare a wide variety of real, three-qubit quantum states. Note that in this preparation, $|000\rangle$ represents the initial all-zero qubit state, $C_{i}^{j}$ is a controlled-NOT (CNOT) gate with $i$ control and $j$ target, $R_{y, i}(\theta)$ is a $\theta$-angle rotation about the $y$ axis of the Bloch sphere on the $i$ th qubit, and $\alpha, \beta, \gamma$, and $\delta$ are variables that depend upon $\theta_{1}, \theta_{2}$, and $\theta_{3}$. By scanning over the angles of rotation $\left(\theta_{1}, \theta_{2}, \theta_{3}\right)$, we prepare states with all possible, real qubit occupation numbers including states that exhibit exciton condensation. We then construct the particle-hole RDM [Eq. (3)] for each preparation by translating the expectation values of the creation and annihilation operators to linear combinations of the expectation values of the Pauli $X, Y, Z$ matrices, which can be directly probed on a quantum device. (See Sec. A 2 in the Appendix for a detailed explanation of this process.) By probing $\lambda_{G}$-the largest eigenvalue of the particle-hole 


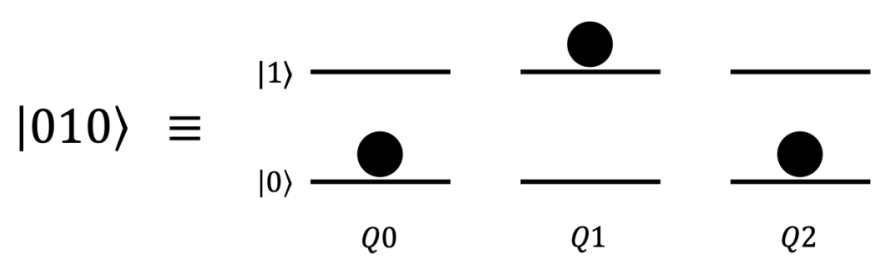

FIG. 1. A schematic demonstrating the interpretation of the $|010\rangle$ state as three $(N)$ particles in six $(2 N)$ orbitals in two triply degenerate ( $N$-degenerate) levels separated by some energetic gap. Each qubit $(Q 0, Q 1, Q 2)$ must contain a particle in either the lower $|0\rangle$ or upper $|1\rangle$ level, and only vertical transitions are allowed.

$\mathrm{RDM}$ - for each of the prepared states, we then determine the extent of exciton condensation for all three-qubit correlation.

Orbital occupation numbers-obtained from the eigenvalues of the one-fermion RDM-are used as a practical coordinate representation in which to visualize $\lambda_{G}$ for all electron correlations (all possible occupations). For a threequbit system, a pure quantum system of three electrons in six orbitals, the requirement that the wave function be antisymmetric for fermionic systems $[35,36]$ further constrains the eigenvalues of the one-particle reduced density matrix (i.e., the orbital occupations) beyond the traditional Pauli constraints $\left(0 \leqslant n_{i} \leqslant 1\right)$ [37]. For a three-qubit quantum system, these relevant so-called generalized Pauli constraints are

$$
n_{5}+n_{6}-n_{4} \geqslant 0 \text {, }
$$

where

$$
\begin{aligned}
& n_{1}+n_{6}=1, \\
& n_{2}+n_{5}=1, \\
& n_{3}+n_{4}=1,
\end{aligned}
$$

in which each $n_{i}$ corresponds the natural-orbital occupations ordered from largest to smallest [38-42]. The three, independent eigenvalues, $n_{4}, n_{5}$, and $n_{6}$, can be used as a threecoordinate representation of a given quantum state against the Pauli polytope, the set of all possible occupations according to the Pauli constraints $\left(0 \leqslant n_{i} \leqslant 1\right)$, as well as the generalized Pauli polytope, the set of all possible occupations according to the generalized Pauli constraint [Eq. (5)] (See Fig. 2).

Scatter plots of the occupation numbers for simulated and mitigated experimental calculations (see Methodology for discussion on error mitigation) are shown in Fig. 2 against the Pauli polytope (the combination of the yellow and blue regions allowed by $\left.0 \leqslant n_{i} \leqslant 1\right)$ and the generalized Pauli polytope [only the yellow region allowed by Eq. (5)]. For the simulated calculations [Fig. 2(a)], possible combinations of angles $\theta_{1}, \theta_{2}$, and $\theta_{3}$, varied systematically for $\theta_{i} \in\left[0, \frac{\pi}{2}\right]$, are used to prepare quantum states according to Eq. (4). Note that the $\lambda_{G}$ value associated with the given calculation is represented by the color of the corresponding sphere in the figure with values increasing from blue to red. As can be seen from Fig. 2, while the preparations span all orbital occupations consistent with the generalized Pauli constraints and hence all electron correlations, only values approaching the $\left(n_{4}, n_{5}, n_{6}\right)=(0.5,0.5,0.5)$ corner of the polytope, known to
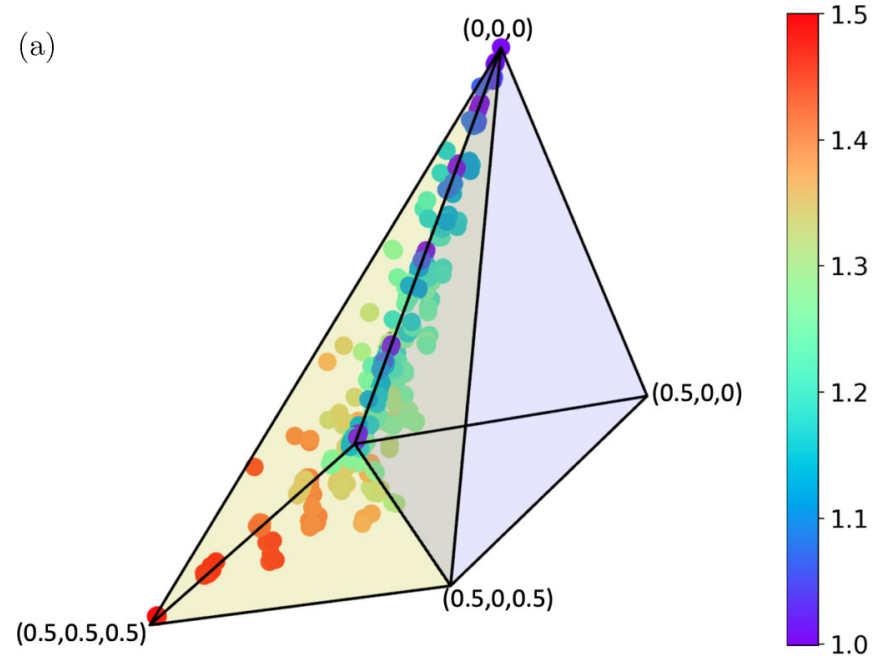

(b)

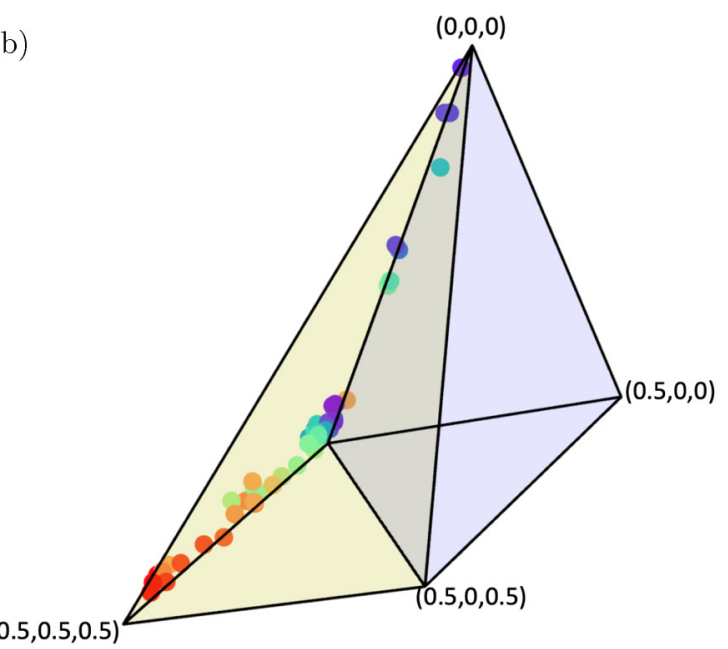

FIG. 2. (a) Simulated and (b) experimental data demonstrating that the occupation numbers $\left(n_{4}, n_{5}, n_{6}\right)$ of the 1-RDM lie in the generalized Pauli polytope (yellow region) with exciton condensate character (with $\lambda_{G}$ increasing from blue to red) emerging as the occupations saturate the vertex $(0.5,0.5,0.5)$.

be the occupations of the GHZ state, demonstrate maximal exciton condensation. The mitigated experimental results shown in Fig. 2(b) in which $\theta_{1}$ is constrained to either 0 or $\frac{\pi}{2}$ to limit computational expense confirm the simulation results.

In order to better visualize the variation of exciton condensate character with respect to variation in the preparation of the qubit quantum state, one particular scan of $\lambda_{G}$ for the minimalistic three-qubit state preparation is shown in Fig. 3 in which the $\theta_{1}$ value is set to zero and the other angles are varied systematically from 0 to $\frac{\pi}{2}$ with a $\frac{\pi}{6}$ interval. In Fig. 3, results are given for (a) simulation, (b) experiments without mitigation, and (c) mitigated experiments. Note that these particular scanning parameters are chosen as they well represent the observed range in $\lambda_{G}$ and demonstrate the maximal three-qubit $\lambda_{G}$ of $\frac{N}{2}=\frac{3}{2}=1.5$ for the simulated results. Additionally, note that even the unmitigated experimental results [(b)] demonstrate a relatively large $\lambda_{G}$ of 1.39 , a clear demonstration of exciton condensate character despite 
(a)

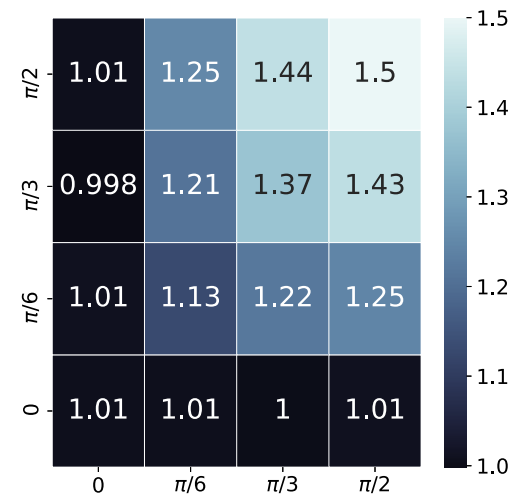

(b)

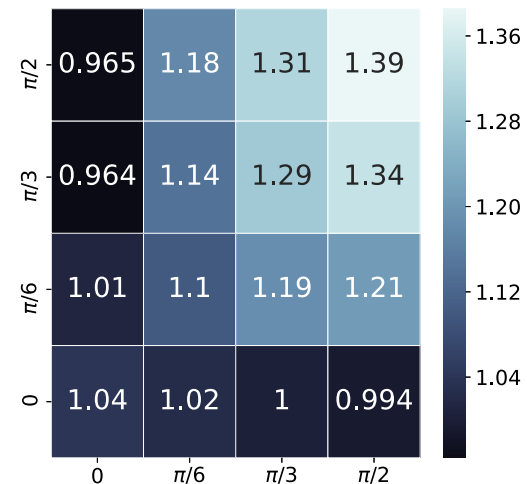

(c)

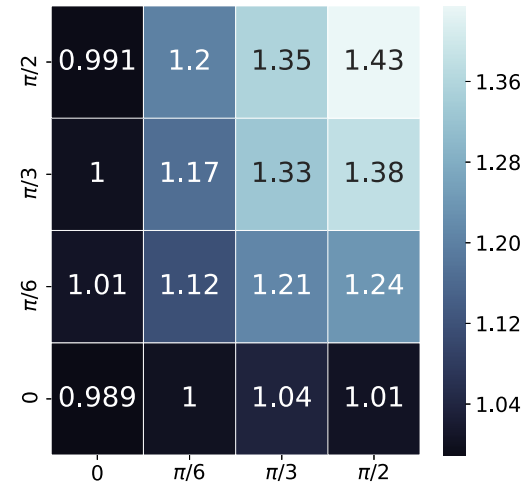

FIG. 3. The largest eigenvalue $\lambda_{G}$ of the modified particle-hole density matrix shown as a function of the preparation angles $\theta_{2}$ and $\theta_{3}$ in the range [0, $\left.\frac{\pi}{2}\right]$ with $\theta_{1}=0$ in Eq. (4) for (a) simulated calculations, (b) experimental results, and (c) mitigated experimental results.

experimental errors (see the Supplemental Material [43] for discussion of errors). This large, non-error-corrected signature of exciton condensation shows that exciton condensation is indeed created on the quantum computer and is not an artifact of error correction. The large eigenvalue $\lambda_{G}$ and the degree of saturation of the generalized Pauli constraint in Eq. (5) are reported in Tables IV through VII and Fig. 1 of the Supplemental Material [43] for many sets of orbital occupations.

\section{B. Condensation with 3 to 53 excitons}

As shown above, the region of the Pauli polytope associated with the GHZ state, the state described by

$$
\left|\Psi_{\mathrm{GHZ}}\right\rangle=\frac{1}{\sqrt{2}}\left(|0\rangle^{\otimes N}+|1\rangle^{\otimes N}\right)
$$

TABLE I. Eigenvalue table for the ${ }^{2} \tilde{G}$ matrix for simulated $\left(\lambda_{G}^{\text {sim }}\right)$, mitigated experimental $\left(\lambda_{G}^{\mathrm{mit}}\right)$, and experimental $\left(\lambda_{G}^{\exp }\right) \mathrm{GHZ}$ state results.

\begin{tabular}{llll}
\hline \hline$N$ & $\lambda_{G}^{\text {sim }}$ & $\lambda_{G}^{\text {mit }}$ & $\lambda_{G}^{\text {exp }}$ \\
\hline 3 & 1.50 & 1.44 & 1.39 \\
4 & 2.00 & 1.92 & 1.80 \\
5 & 2.50 & 2.33 & 2.22 \\
6 & 3.00 & 2.70 & 2.27 \\
7 & 3.50 & & 2.44 \\
8 & 4.00 & & 2.72 \\
9 & 4.50 & & 2.73 \\
10 & 5.00 & & 2.93 \\
11 & 5.50 & & 3.08 \\
12 & 6.00 & & 3.22 \\
13 & 6.50 & & 3.28 \\
14 & 7.00 & & 3.25 \\
15 & 7.50 & & $2.91^{\mathrm{a}}$ \\
16 & 8.00 & & 2.28 \\
22 & 11.0 & & 2.68 \\
28 & 14.0 & & 3.48 \\
34 & 17.0 & & 3.82 \\
41 & 20.5 & & 3.99 \\
47 & 23.5 & & $4.74^{\mathrm{a}}$ \\
53 & 26.5 & & \\
\hline \hline
\end{tabular}

${ }^{\text {a No suitable circuit orientation on quantum device for creation of }}$ a 15-qubit or 53-qubit GHZ state, introducing excess error to the calculation.

for an $\mathrm{N}$-qubit system, demonstrates maximal exciton condensate character for three qubits. While the computations with maximal exciton condensation have occupation numbers consistent with the GHZ state (among other states), the minimalistic preparation used to probe $\lambda_{G}$ in the previous section permits only double excitations as shown in Eq. (4). As the GHZ state is relatively easily generalizable to higher-qubit preparations and is hence desirable, a different qubit preparation scheme [Eq. (A4) in the Appendix] is used to generate the three-qubit GHZ state and verify that the true GHZ state demonstrates exciton condensation. As can be seen in Table I, maximum exciton condensate character $\left(\frac{N}{2}=\frac{3}{2}=1.5\right)$ is indeed observed for simulation of the three-qubit GHZ state.

The generalizable qubit preparation of the GHZ for a generic $N$-qubit state allows for the extension of the above result to larger numbers of qubits, the outcomes of which can be seen in Table I. These results demonstrate that the beginning of exciton condensation is achieved on quantum computers using 3 to 53 qubits. Note that error mitigation is limited to systems with $N \leqslant 6$ qubits as larger-qubit error mitigation schemes necessitate more expensive circuits for the quantum device. Additionally, to limit computational expense, only real contributions to the reduced density matrices are computed. See the Appendix for specific experimental details and Tables I, II, and III of the Supplemental Material [43] for device specifications.

As is apparent from simulated results [Table I and Fig. 4(a)], the GHZ state for all qubits yields the maximal value for the exciton condensate character of $\frac{N}{2}$. As such, the GHZ state is expected to demonstrate maximal exciton 
(a)

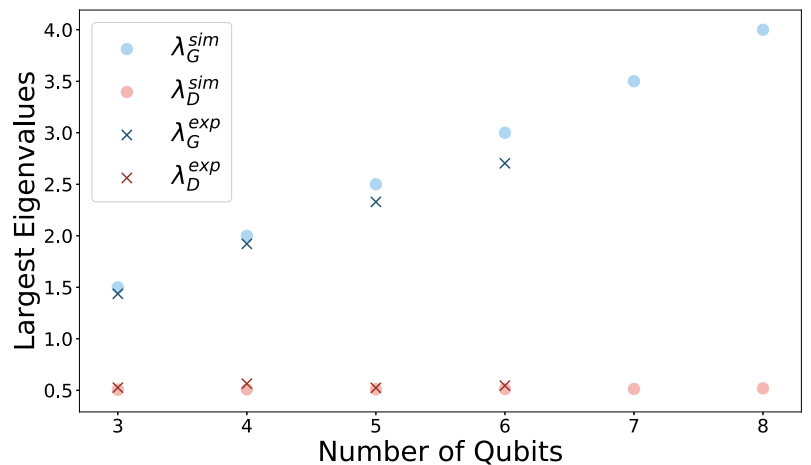

(b)

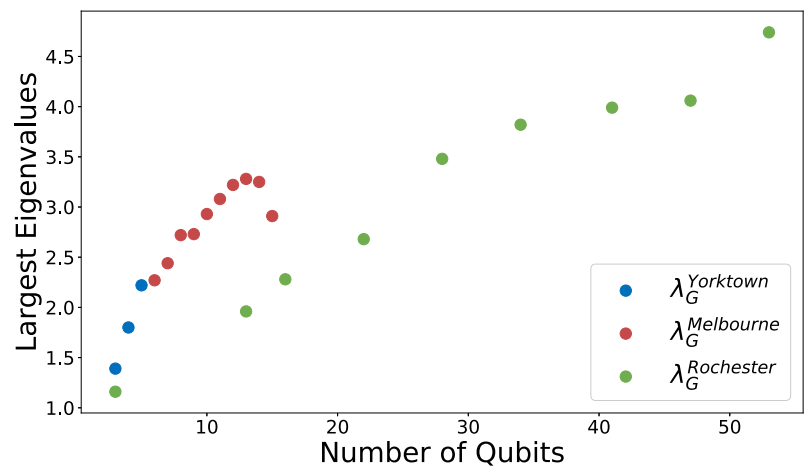

(c)

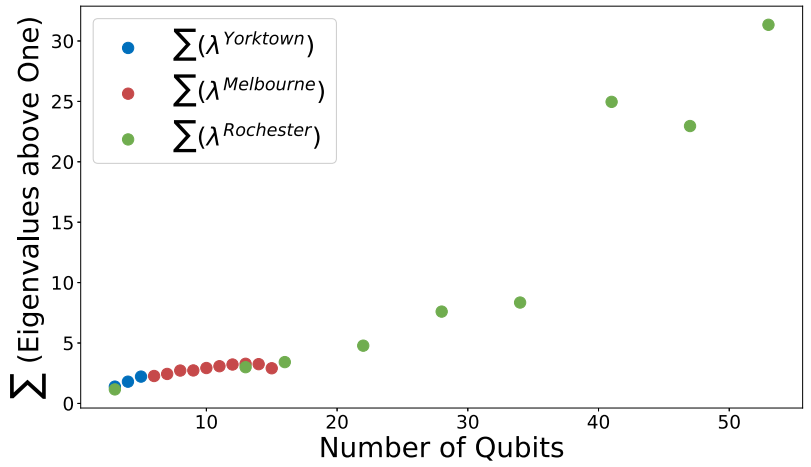

FIG. 4. Plots showing (a) the largest eigenvalue of the ${ }^{2} \tilde{G}$ matrix (blue) and the ${ }^{2} D$ matrix (red) for simulated (dots) and all mitigated experimental ( $\times$ 's) calculations, (b) the largest eigenvalue of the unmitigated experimental ${ }^{2} \tilde{G}$ matrix, and (c) the sum of all eigenvalues of the unmitigated experimental ${ }^{2} \tilde{G}$ matrix demonstrating exciton condensation $(\lambda>1)$ for experiments of $N$ qubits on the Yorktown 5-qubit IBM Quantum Experience device (blue), the Melbourne 15-qubit IBM Quantum Experience device (red), and the Rochester 53-qubit IBM Quantum Experience device (green).

condensation for a given number of qubits. While the experimental results in Table I and Fig. 4(b) do not achieve maximal $\lambda_{G}$ values, although the error-mitigated results do appear to approach $\frac{N}{2}$, exciton condensation character $\left(\lambda_{G}>1\right)$ is observed for each GHZ state prepared for $N=3$ to 53 qubits.

The larger deviation from the simulated results observed in the higher-qubit experiments-in which there seems to be a maximal signature of exciton condensation of around $\lambda_{G} \approx 3$ [Table I and Fig. 4(b)] - is likely due to the cumulative effects of errors that become increasingly significant as the number of qubits - and hence the number of CNOT gates appliedincreases. (See the Supplemental Material [43] for details of gate errors, readout errors, and multiqubit CNOT errors for the quantum devices employed for experimentation.) These errors seem to prevent the formation of a global excitonic state due to dispersion; however, as the number of qubits is increased, the condensation behavior of the $N$-qubit system does still increase as is shown in Fig. 4(c). In these higherqubit experiments, there are multiple eigenvalues of the ${ }^{2} \tilde{G}$ matrix above 1, indicating that there are multiple eigenstates demonstrating character of exciton condensation. The sum of the eigenvalues above 1 increases in an almost linear fashion as the number of qubits is increased, demonstrating an overall increase in the excitonic nature of the prepared states even if maximal condensation behavior in a single orbital cannot be obtained for these higher-qubit experiments due to dispersion.

The GHZ state is often referred to as a "the maximally entangled state" as it has maximum entanglement entropy [44]; however, there are diverse types of nonequivalent multipartite entanglement. For example, Bose-Einstein condensation, fermion pair condensation, and exciton condensation are all phenomena that occur due to the entanglement of bosons, differing in their signatures and the types of bosons that are entangled. Here we have demonstrated a new characteristic of the maximal entanglement of the GHZ state-namely the maximal entanglement of particle-hole pairs (excitons). Further, the fermion pair condensate character $\left(\lambda_{D}\right)$ is additionally probed for the GHZ state, and no fermion pair condensation is observed $\left(\lambda_{D}<1\right)$. (See Fig. 4.) As such, we have shown that the maximal entanglement of the GHZ state does not correspond to the entanglement of particle-particle pairing.

\section{DISCUSSION AND CONCLUSIONS}

In this study, we have prepared molecular-scale exciton condensates for 3- to 53-qubit systems on three quantum computers and verified the presence of the condensation through postmeasurement computation of the exciton condensate's quantum signature [24]. The maximal condensate character is observed for the Greenberger-Horne-Zeilinger (GHZ) "maximally entangled" state, indicating that a characteristic of this maximally entangled state is the entanglement of particlehole pairs (excitons). Further, as transmon qubit quantum states [1,3-9] are experimentally constructed on the quantum devices employed, these exciton condensates corresponding to the GHZ state can be interpreted as exciton condensates of photons - the entanglement of photon-hole pairs. Whether photon-hole pair condensation would have similar properties to those of traditional fermion-hole exciton condensates is unknown, but it seems likely that, as the photons are directly analogous to fermions in a traditional exciton condensate, the superfluidity of photon-hole excitons should allow for the dissipationless flow of energy, which has possible applications in energy transport.

Additionally, the recognition of the GHZ state as an exciton condensate on a quantum computer establishes a new avenue for the creation and characterization of exciton condensates. As the GHZ state can be remotely and reliably constructed and probed through the use of cloud-accessible quantum devices, this preparation of exciton condensation may be more convenient than prior experimental methodologies. Moreover, multiparticle GHZ states have previously been experimentally 
realized through optical methodologies [45-47], ion traps [48], and even Ising spin models [49], demonstrating that various types of exciton condensates can be-and indeed have been-prepared. The recognition that such established methodologies for preparation of the GHZ state also demonstrate exciton condensation may advance the search for the creation of convenient, high-temperature, ground state exciton condensation, which may have technological applications.

As quantum devices are created with a larger number of qubits, preparation of these higher-qubit GHZ states would create more macroscopically scaled exciton condensates of these various compositions, although - as we have demonstrated-unless sufficient effort is done to prevent dispersion, this condensate character will be scattered throughout multiple eigenstates of the particle-hole RDM. Thus, future exploration of the properties of exciton condensates on quantum computers is anticipated, and the creation and characterization of exciton condensates is yet another motivation for the development of low-error quantum devices with macroscopic numbers of qubits.

\section{ACKNOWLEDGMENTS}

The authors acknowledge use of the IBM Quantum Experience devices for this work. D.A.M. gratefully acknowledges the Department of Energy, Office of Basic Energy Sciences, Grant No. DE-SC0019215, the US National Science Foundation, Grants No. CHE-1565638, No. CHE-2035876, and No. DMR-2037783, and the US Army Research Office (ARO), Grant No. W911NF-16-1-0152. The views expressed are those of the authors and do not reflect the official policy or position of IBM or the IBM Q team.

D.A.M. conceived the project. L.M.S. performed the computations on the quantum computers. L.M.S., S.E.S., and D.A.M. analyzed the data and wrote the manuscript.

The authors do not have competing interests.

\section{APPENDIX}

We include details on the quantum algorithms used to prepare the qubit states presented in the article; the quantum tomography of the modified particle-hole reduced density matrix; the methodology by which error is mitigated; and relevant details on the experimental quantum devices employed.

\section{State preparations}

Two algorithms are utilized in this work to prepare the qubit states.

Minimalistic scanning approach. The first algorithm takes a minimalistic approach to span all valid one-qubit occupation numbers for a three-qubit system and is given as follows:

$$
|\Psi\rangle=C_{3}^{2} R_{y, 3}\left(\theta_{3}\right) C_{1}^{2} R_{y, 1}\left(\theta_{2}\right) C_{1}^{3} R_{y, 1}\left(\theta_{1}\right)|0\rangle^{\otimes 3},
$$

where $R_{y, i}$ refers to rotation of a qubit $i$ about its Bloch sphere's $y$ axis, which is given by

$$
R_{y, i}(\theta)=\left(\begin{array}{rr}
\cos \left(\frac{\theta}{2}\right) & -\sin \left(\frac{\theta}{2}\right) \\
\sin \left(\frac{\theta}{2}\right) & \cos \left(\frac{\theta}{2}\right)
\end{array}\right),
$$

and $C_{i}^{j}$ is a standard controlled-NOT (CNOT) gate with control and target qubits $i$ and $j$, respectively. Note that the control qubit is rotated prior to the application of the CNOT transformation. Overall, the sequence of transformations in Eq. (A1) yields a wave function of the form

$$
|\Psi\rangle=\alpha|000\rangle+\beta|011\rangle+\gamma|101\rangle+\delta|110\rangle,
$$

such that $\alpha, \beta, \gamma$, and $\delta$ are functions of the input angles $\left(\theta_{1}, \theta_{2}, \theta_{3}\right)$ and the 1-RDM contains solely diagonal elements.

The GHZ state. The GHZ state described in Eq. (9) is prepared according to

$$
|\Psi\rangle=C_{N-1}^{N} \cdots C_{2}^{3} C_{1}^{2} H_{1}|0\rangle^{\otimes N}
$$

for an $N$-qubit state where $H_{i}$ represents the Hadamard gatewhich maps $|0\rangle$ to $\frac{|0\rangle+|1\rangle}{\sqrt{2}}$ and $|1\rangle$ to $\frac{|0\rangle-|1\rangle}{\sqrt{2}}$ - acting on qubit $i$. There has been much study on the optimal preparation of the GHZ state for various numbers of qubits [50-53]; in this study, however, the simple algorithm from Ref. [54] for GHZ state preparation is utilized as it is easily implemented and generalizable to any arbitrary number of qubits.

\section{Quantum tomography of the particle-hole RDM}

The modified particle-hole RDM-with elements given by Eq. (3) - is obtained through the translation of all of its elements into the bases of Pauli matrices, which are directly probed on the quantum computer.

First, let us focus on the ${ }^{1} D_{i}^{j}$ terms of the ${ }^{2} \tilde{G}$ matrix elements. As 1-RDMs simplify to block-diagonal forms with respect to single qubits, there are no nonzero two-qubit 1RDM terms. In order for a 1-RDM to be nonzero, then, it must be a one-qubit 1-RDM of the form

$$
\begin{array}{c|cc} 
& \hat{a}_{p, 0} & \hat{a}_{p, 1} \\
\hline \hat{a}_{p, 0}^{\dagger} & \hat{a}_{p, 0}^{\dagger} \hat{a}_{p, 0} & \hat{a}_{p, 0}^{\dagger} \hat{a}_{p, 1} \\
\hat{a}_{p, 1}^{\dagger} & \hat{a}_{p, 1}^{\dagger} \hat{a}_{p, 0} & \hat{a}_{p, 1}^{\dagger} \hat{a}_{p, 1}
\end{array}
$$

for qubit $p$ where $\hat{a}_{p}^{\dagger}$ and $\hat{a}_{p}$ are creation and annihilation operators for qubit $p$, respectively. Each term of these nonzero, one-qubit 1-RDMs can be written as a linear combination of Pauli matrices. For example, $\hat{a}_{p, 0}^{\dagger} \hat{a}_{p, 1}$, which represents the qubit going from state $|1\rangle=\left(\begin{array}{l}0 \\ 1\end{array}\right)$ to state $|0\rangle=\left(\begin{array}{l}1 \\ 0\end{array}\right)$, can be written as follows:

$$
\hat{a}_{p, 0}^{\dagger} \hat{a}_{p, 1}=\left(\begin{array}{ll}
0 & 1 \\
0 & 0
\end{array}\right)=\frac{1}{2}\left(X_{p}+i Y_{p}\right)
$$

Similarly, the other elements can be represented as shown:

$$
\begin{gathered}
\hat{a}_{p, 1}^{\dagger} \hat{a}_{p, 0}=\left(\begin{array}{ll}
0 & 0 \\
1 & 0
\end{array}\right)=\frac{1}{2}\left(X_{p}-i Y_{p}\right), \\
\hat{a}_{p, 0}^{\dagger} \hat{a}_{p, 0}=\left(\begin{array}{ll}
1 & 0 \\
0 & 0
\end{array}\right)=\frac{1}{2}\left(I+Z_{p}\right), \\
\hat{a}_{p, 1}^{\dagger} \hat{a}_{p, 1}=\left(\begin{array}{ll}
0 & 0 \\
0 & 1
\end{array}\right)=\frac{1}{2}\left(I-Z_{p}\right) .
\end{gathered}
$$

(See the Supplemental Material [43] for a discussion on how our representation of creation and annihilation operators corresponds to bosonic and fermionic statistics.) The expectation value of each matrix element for a given qubit $(p)$ can then be obtained by directly probing the expectation values of $X_{p}, Y_{p}$, and $Z_{p}$ for a given state preparation. 
The overall particle-hole RDM $\left({ }^{2} G\right.$ matrix $)$ can be represented as a $4 N \times 4 N$ matrix composed of $N^{2} 4 \times 4$ submatrices of the form

\begin{tabular}{c|cccc} 
& $\hat{a}_{q, 0}^{\dagger} \hat{a}_{q, 0}$ & $\hat{a}_{q, 1}^{\dagger} \hat{a}_{q, 0}$ & $\hat{a}_{q, 0}^{\dagger} \hat{a}_{q, 1}$ & $\hat{a}_{q, 1}^{\dagger} \hat{a}_{q, 1}$ \\
\hline$\hat{a}_{p, 0}^{\dagger} \hat{a}_{p, 0}$ & $\hat{a}_{p, 0}^{\dagger} \hat{a}_{p, 0} \hat{a}_{q, 0}^{\dagger} \hat{a}_{q, 0}$ & $\hat{a}_{p, 0}^{\dagger} \hat{a}_{p, 0} \hat{a}_{q, 1}^{\dagger} \hat{a}_{q, 0}$ & $\hat{a}_{p, 0}^{\dagger} \hat{a}_{p, 0} \hat{a}_{q, 0}^{\dagger} \hat{a}_{q, 1}$ & $\hat{a}_{p, 0}^{\dagger} \hat{a}_{p, 0} \hat{a}_{q, 1}^{\dagger} \hat{a}_{q, 1}$ \\
$\hat{a}_{p, 0}^{\dagger} \hat{a}_{p, 1}$ & $\hat{a}_{p, 0}^{\dagger} \hat{a}_{p, 1} \hat{a}_{q, 0}^{\dagger} \hat{a}_{q, 0}$ & $\hat{a}_{p, 0}^{\dagger} \hat{a}_{p, 1} \hat{a}_{q, 1}^{\dagger} \hat{a}_{q, 0}$ & $\hat{a}_{p, 0}^{\dagger} \hat{a}_{p, 1} \hat{a}_{q, 0}^{\dagger} \hat{a}_{q, 1}$ & $\hat{a}_{p, 0}^{\dagger} \hat{a}_{p, 1} \hat{a}_{q, 1}^{\dagger} \hat{a}_{q, 1}$ \\
$\hat{a}_{p, 1}^{\dagger} \hat{a}_{p, 0}$ & $\hat{a}_{p, 1}^{\dagger} \hat{a}_{p, 0} \hat{a}_{q, 0}^{\dagger} \hat{a}_{q, 0}$ & $\hat{a}_{p, 1}^{\dagger} \hat{a}_{p, 0} \hat{a}_{q, 1}^{\dagger} \hat{a}_{q, 0}$ & $\hat{a}_{p, 1}^{\dagger} \hat{a}_{p, 0} \hat{a}_{q, 0}^{\dagger} \hat{a}_{q, 1}$ & $\hat{a}_{p, 1}^{\dagger} \hat{a}_{p, 0} \hat{a}_{q, 1}^{\dagger} \hat{a}_{q, 1}$ \\
$\hat{a}_{p, 1}^{\dagger} \hat{a}_{p, 1}$ & $\hat{a}_{p, 1}^{\dagger} \hat{a}_{p, 1} \hat{a}_{q, 0}^{\dagger} \hat{a}_{q, 0}$ & $\hat{a}_{p, 1}^{\dagger} \hat{a}_{p, 1} \hat{a}_{q, 1}^{\dagger} \hat{a}_{q, 0}$ & $\hat{a}_{p, 1}^{\dagger} \hat{a}_{p, 1} \hat{a}_{q, 0}^{\dagger} \hat{a}_{q, 1}$ & $\hat{a}_{p, 1}^{\dagger} \hat{a}_{p, 1} \hat{a}_{q, 1}^{\dagger} \hat{a}_{q, 1}$
\end{tabular}

where each element of the matrix is the expectation value of the creation and annihilation operator terms shown. As multiqubit wave functions are the tensor products of individual qubit wave functions, these four-body terms can be represented as the tensor products of the two-body terms composing them. For example, the matrix element given by $\hat{a}_{p, 0}^{\dagger} \hat{a}_{p, 1} \hat{a}_{q, 1}^{\dagger} \hat{a}_{q, 1}$ can be written as

$$
\hat{a}_{p, 0}^{\dagger} \hat{a}_{p, 1} \hat{a}_{q, 1}^{\dagger} \hat{a}_{q, 1}=\left(\hat{a}_{p, 0}^{\dagger} \hat{a}_{p, 1}\right) \otimes\left(\hat{a}_{q, 1}^{\dagger} \hat{a}_{q, 1}\right)=\left[\frac{1}{2}\left(X_{p}+i Y_{p}\right)\right] \otimes\left[\frac{1}{2}\left(\hat{I}_{q}-Z_{q}\right)\right]=\frac{1}{4}\left[X_{p} \otimes \hat{I}_{q}-X_{p} \otimes Z_{q}+i Y_{p} \otimes \hat{I}_{q}-i Y_{p} \otimes Z_{q}\right],
$$

where $Y_{p} \otimes Z_{q}$ is one of nine possible two-qubit tensor products. The Pauli representation of all other matrix elements can be determined using analogous, straightforward methodologies, and the values of these matrix elements are then calculated by probing the expectation values of the two-qubit tensor products of Pauli matrices.

Similarly, the overall modified particle-hole $\operatorname{RDM}\left({ }^{2} \tilde{G}\right.$ matrix) can be represented as a $4 N \times 4 N$ matrix composed of $N^{2} 4 \times 4$ submatrices. These submatrices are identical to the submatrices of the ${ }^{2} G$ matrix with the block modification shown below subtracted off to eliminate the extraneous ground state to ground state transition:

\begin{tabular}{c|cccc} 
& $\hat{a}_{q, 0}^{\dagger} \hat{a}_{q, 0}$ & $\hat{a}_{q, 1}^{\dagger} \hat{a}_{q, 0}$ & $\hat{a}_{q, 0}^{\dagger} \hat{a}_{q, 1}$ & $\hat{a}_{p, 1}^{\dagger} \hat{a}_{p, 1}$ \\
\hline$\hat{a}_{p, 0}^{\dagger} \hat{a}_{p, 0}$ & ${ }^{1} D_{p}[0,0]{ }^{1} D_{q}[0,0]$ & ${ }^{1} D_{p}[0,0]{ }^{1} D_{q}[0,1]$ & ${ }^{1} D_{p}[0,0]{ }^{1} D_{q}[1,0]$ & ${ }^{1} D_{p}[0,0]{ }^{1} D_{q}[1,1]$ \\
$\hat{a}_{p, 0}^{\dagger} \hat{a}_{p, 1}$ & ${ }^{1} D_{p}[0,1]{ }^{1} D_{q}[0,0]$ & ${ }^{1} D_{p}[0,1]{ }^{1} D_{q}[0,1]$ & ${ }^{1} D_{p}[0,1]{ }^{1} D_{q}[1,0]$ & ${ }^{1} D_{p}[0,1]{ }^{1} D_{q}[1,1]$ \\
$\hat{a}_{p, 1}^{\dagger} \hat{a}_{p, 0}$ & ${ }^{1} D_{p}[1,0]{ }^{1} D_{q}[0,0]$ & ${ }^{1} D_{p}[1,0]{ }^{1} D_{q}[0,1]$ & ${ }^{1} D_{p}[1,0]{ }^{1} D_{q}[1,0]$ & ${ }^{1} D_{p}[1,0]{ }^{1} D_{q}[1,1]$ \\
$\hat{a}_{p, 1}^{\dagger} \hat{a}_{p, 1}$ & ${ }^{1} D_{p}[1,1]{ }^{1} D_{q}[0,0]$ & ${ }^{1} D_{p}[1,1]{ }^{1} D_{q}[0,1]$ & ${ }^{1} D_{p}[1,1]{ }^{1} D_{q}[1,0]$ & ${ }^{1} D_{p}[1,1]{ }^{1} D_{q}[1,1]$
\end{tabular}

Note that ${ }^{1} D_{i}$ is the RDM for qubit $i$ described in Eq. (A5) and that ${ }^{1} D_{i}[a, b]$ is the element of that matrix with matrix coordinates $[a, b]$.

The overall form of the ${ }^{2} \tilde{G}$ matrix is hence

\begin{tabular}{|c|c|c|c|}
\hline$p=0, q=0$ & $p=0, q=1$ & $\cdots$ & $p=0, q=N-1$ \\
\hline$p=1, q=0$ & $p=1, q=1$ & $\cdots$ & $p=1, q=N-1$ \\
\hline$\vdots$ & $\vdots$ & $\ddots$ & $\vdots$ \\
\hline$p=N-1, q=0$ & $p=N-1, q=1$ & $\cdots$ & $p=N-1, q=N-1$ \\
\hline
\end{tabular}

where each $p / q$ combination represents one of the previously specified blocks, i.e., the difference of the matrices given in Eqs. (A10) and (A12). The largest eigenvalue of this overall matrix is the $\lambda_{G}$ value employed throughout this article.

In order to probe the extent of fermion pair condensation, the particle-particle RDM shown in Eq. (1) must additionally be constructed. Similarly to both the ${ }^{2} G$ and ${ }^{2} \tilde{G}$ matrices, the ${ }^{2} D$ matrix can be represented as a $4 N \times 4 N$ matrix, and the elements of the ${ }^{2} D$ matrix can be computed according to

$$
{ }^{2} D_{k, l}^{i, j}=\delta_{l}^{j}{ }^{1} D_{k}^{i}-{ }^{2} G_{k, j}^{i, l},
$$

where $\delta_{l}^{j}$ is a delta function whose value is defined to be 1 when $i=j$ and otherwise zero and where ${ }^{1} D_{k}^{i}$ and ${ }^{2} G_{k, j}^{i, l}$ are the one-particle RDM and the particle-hole RDM whose elements are computed according to Eqs. (A5)-(A9) and Eqs. (A10) and (A11), respectively. The signature of fermion pair condensation-i.e., $\lambda_{D}$, the largest eigenvalue of the ${ }^{2} D$ matrix - can then be obtained from the particle-particle RDM obtained for a given preparation.

As the states prepared in this study are real wave functions, the imaginary components of the RDMs should be approximately zero within a small range dictated by inherent randomness and by the error of the devices. Therefore, only the five of the possible nine two-qubit expectation values that correspond to real contributions to the RDMs $\left[\left\langle X_{p} \otimes X_{q}\right\rangle\right.$, $\left\langle Y_{p} \otimes Y_{q}\right\rangle,\left\langle Z_{p} \otimes Z_{q}\right\rangle,\left\langle X_{p} \otimes Z_{q}\right\rangle$, and $\left.\left\langle Z_{p} \otimes X_{q}\right\rangle\right]$ are nonzero and hence essential for construction of the ${ }^{2} \tilde{G}$ matrix. While, for the sake of completeness, the negligibly small imaginary components are included in the construction of the ${ }^{2} \tilde{G}$ matrix for the low-qubit $(N=3-5)$ computations, only real components are included in the ${ }^{2} \tilde{G}$ matrix for higherqubit $(N=6-15)$ computations to lower computational expense.

Error mitigation. A measurement correction fitter for a tensored calibration is employed to mitigate measurement error through use of the "least-squares" method - which constrains the resultant mitigated counts to having physical 
probabilities- to construct a mitigation filter that can be applied to experimental data $[55,56]$.

\section{Quantum device specifications}

Throughout this work, we employ the ibmqx2 (ibmq_5_yorktown) [57], the ibmq_16_melbourne [58], and the ibmq_rochester [59] IBM Quantum Experience devices, which are available online. Unless explicitly stated otherwise, all low-qubit $(N \leqslant 5)$ experiments are conducted using the 5-qubit ibmqx2 (ibmq_5_yorktown) device, all midrange-qubit $(5<N \leqslant 15)$ experiments are conducted using the 15-qubit ibmq_16_melbourne device, and all high-qubit $(N>15)$ experiments are conducted using the 53-qubit ibmq_rochester device. These quantum devices are composed of fixed-frequency transmon qubits with coplanar waveguide resonators [15,17]. Experimental calibration data and connectivity for these devices are included in the Supplemental Material [43].
[1] D. V. Fil and S. I. Shevchenko, Electron-hole superconductivity, Low Temp. Phys. 44, 867 (2018).

[2] L. V. Keldysh, Coherent states of excitons, Phys. Usp. 60, 1180 (2017).

[3] M. Kellogg, J. P. Eisenstein, L. N. Pfeiffer, and K. W. West, Vanishing Hall Resistance at High Magnetic Field in A DoubleLayer Two-Dimensional Electron System, Phys. Rev. Lett. 93, 036801 (2004).

[4] E. Tutuc, M. Shayegan, and D. A. Huse, Counterflow Measurements in Strongly Correlated GaAs Hole Bilayers: Evidence for Electron-Hole Pairing, Phys. Rev. Lett. 93, 036802 (2004).

[5] S. Safaei and D. A. Mazziotti, Quantum signature of exciton condensation, Phys. Rev. B 98, 045122 (2018).

[6] A. Kogar, M. S. Rak, S. Vig, A. A. Husain, F. Flicker, Y. I. Joe, L. Venema, G. J. MacDougall, T. C. Chiang, E. Fradkin, J. van Wezel, and P. Abbamonte, Signatures of exciton condensation in a transition metal dichalcogenide, Science 358, 1314 (2017).

[7] X. Liu, K. Watanabe, T. Taniguchi, B. I. Halperin, and P. Kim, Quantum Hall drag of exciton condensate in graphene, Nat. Phys. 13, 746 (2017).

[8] D. Varsano, S. Sorella, D. Sangalli, M. Barborini, S. Corni, E. Molinari, and M. Rontani, Carbon nanotubes as excitonic insulators, Nat. Commun. 8, 1461 (2017).

[9] M. S. Fuhrer and A. R. Hamilton, Chasing the exciton condensate, Physics 9, 80 (2016).

[10] J. Kasprzak, M. Richard, S. Kundermann, A. Baas, P. Jeambrun, J. M. J. Keeling, F. M. Marchetti, M. H. Szymanska, R. André, J. L. Staehli, V. Savona, P. B. Littlewood, B. Deveaud, and Le Si Dang, Bose-Einstein condensation of exciton polaritons, Nature (London) 443, 409 (2006).

[11] I. B. Spielman, J. P. Eisenstein, L. N. Pfeiffer, and K. W. West, Resonantly Enhanced Tunneling in a Double Layer Quantum Hall Ferromagnet, Phys. Rev. Lett. 84, 5808 (2000).

[12] D. Nandi, A. D. K. Finck, J. P. Eisenstein, L. N. Pfeiffer, and K. W. West, Exciton condensation and perfect Coulomb drag, Nature (London) 488, 481 (2012).

[13] R. Ma, B. Saxberg, C. Owens, N. Leung, Y. Lu, J. Simon, and D. I. Schuster, A dissipatively stabilized Mott insulator of photons, Nature (London) 566, 51 (2019).

[14] A. Wallraff, D. I. Schuster, A. Blais, L. Frunzio, R.-S. Huang, J. Majer, S. Kumar, S. M. Girvin, and R. J. Schoelkopf, Strong coupling of a single photon to a superconducting qubit using circuit quantum electrodynamics, Nature (London) 431, 162 (2004).

[15] J. Koch, T. M. Yu, J. Gambetta, A. A. Houck, D. I. Schuster, J. Majer, A. Blais, M. H. Devoret, S. M. Girvin, and R. J.
Schoelkopf, Charge-insensitive qubit design derived from the Cooper pair box, Phys. Rev. A 76, 042319 (2007).

[16] M. Göppl, A. Fragner, M. Baur, R. Bianchetti, S. Filipp, J. M. Fink, P. J. Leek, G. Puebla, L. Steffen, A. Wallraff et al., Coplanar waveguide resonators for circuit quantum electrodynamics, J. Appl. Phys. 104, 113904 (2008)

[17] J. M. Chow, A. D. Córcoles, J. M. Gambetta, C. Rigetti, B. R. Johnson, J. A. Smolin, J. R. Rozen, G. A. Keefe, M. B. Rothwell, M. B. Ketchen et al., Simple All-Microwave Entangling Gate for Fixed-Frequency Superconducting Qubits, Phys. Rev. Lett. 107, 080502 (2011).

[18] G. Andersson, Circuit quantum electrodynamics with a transmon qubit in a 3D cavity, master's thesis, Walther Meißner Institut, 2015.

[19] H. J. Lipkin, N. Meshkov, and A. J. Glick, Validity of manybody approximation methods for a solvable model. I. Exact solutions and perturbation theory, Nucl. Phys. A 62, 188 (1965).

[20] R. Pérez, M. C. Cambiaggio, and J. P. Vary, $t$ expansion and the Lipkin model, Phys. Rev. C 37, 2194 (1988).

[21] D. A. Mazziotti, Contracted Schrödinger equation: Determining quantum energies and two-particle density matrices without wave functions, Phys. Rev. A 57, 4219 (1998).

[22] J. Stein, Unitary flow of the bosonized large- $N$ Lipkin model, J. Phys. G: Nucl. Part. Phys. 26, 377 (2000).

[23] D. A. Mazziotti, Exactness of wave functions from two-body exponential transformations in many-body quantum theory, Phys. Rev. A 69, 012507 (2004).

[24] C. Garrod and M. Rosina, Particle-hole matrix: Its connection with the symmetries and collective features of the ground state, J. Math. Phys. 10, 1855 (1969).

[25] S. N. Bose and A. Einstein, Planck's law and light quantum hypothesis, Z. Phys. 26, 178 (1924).

[26] A. Einstein, Quantentheorie des einatomigen idealen Gases, in Albert Einstein: Akademie-Vorträge, edited by D. Simon (Wiley Online Library, Berlin, Germany, 2006), https://onlinelibrary. wiley.com/doi/abs/10.1002/3527608958.ch27.

[27] F. London, On Bose-Einstein condensation, Phys. Rev. 54, 947 (1938).

[28] L. Tisza, The theory of liquid helium, Phys. Rev. 72, 838 (1947).

[29] W. Pauli, The connection between spin and statistics, Phys. Rev. 58, 716 (1940).

[30] P. W. Anderson, Twenty-five years of high-temperature superconductivity: A personal review, J. Phys.: Conf. Ser. 449 , 012001 (2013).

[31] C. N. Yang, Concept of off-diagonal long-range order and the quantum phases of liquid $\mathrm{He}$ and of superconductors, Rev. Mod. Phys. 34, 694 (1962). 
[32] F. Sasaki, Eigenvalues of fermion density matrices, Phys. Rev. 138, B1338 (1965).

[33] W. Kohn and D. Sherrington, Two kinds of bosons and Bose condensates, Rev. Mod. Phys. 42, 1 (1970).

[34] S. E. Smart, D. I. Schuster, and D. A. Mazziotti, Experimental data from a quantum computer verifies the generalized Pauli exclusion principle, Commun. Phys. 2, 11 (2019).

[35] W. Heisenberg, Mehrkörperproblem und resonanz in der quantenmechanik, Z. Phys. 38, 411 (1926).

[36] P. A. M. Dirac, The physical interpretation of the quantum dynamics, Proc. R. Soc. London 113, 621 (1927).

[37] R. E. Borland and K. Dennis, The conditions on the one-matrix for three-body fermion wavefunctions with one-rank equal to six, J. Phys. B 5, 7 (1972).

[38] C. Schilling, D. Gross, and M. Christandl, Pinning of Fermionic Occupation Numbers, Phys. Rev. Lett. 110, 040404 (2013).

[39] C. L. Benavides-Riveros, J. M. Gracia-Bondía, and M. Springborg, Quasipinning and entanglement in the lithium isoelectronic series, Phys. Rev. A 88, 022508 (2013).

[40] R. Chakraborty and D. A. Mazziotti, Generalized Pauli conditions on the spectra of one-electron reduced density matrices of atoms and molecules, Phys. Rev. A 89, 042505 (2014).

[41] D. A. Mazziotti, Pure- $N$-representability conditions of twofermion reduced density matrices, Phys. Rev. A 94, 032516 (2016).

[42] D. A. Mazziotti, Structure of Fermionic Density Matrices: Complete $N$-Representability Conditions, Phys. Rev. Lett. 108, 263002 (2012).

[43] See Supplemental Material at http://link.aps.org/supplemental/ 10.1103/PhysRevResearch.2.043205 for the specifications of the 53-qubit quantum computer, detailed orbital occupations and their pinning to generalized Pauli constraints, as well as a discussion of qubit representations of bosons and fermions.

[44] M. Walter, D. Gross, and J. Eisert, Multipartite entanglement, in Quantum Information, edited by D. Bruß and G. Leuchs (Wiley Online Library, Weinheim, Germany, 2016), https://doi.org/10. 1002/9783527805785.ch14.

[45] D. Bouwmeester, J.-W. Pan, M. Daniell, H. Weinfurter, and A. Zeilinger, Observation of Three-Photon GreenbergerHorne-Zeilinger Entanglement, Phys. Rev. Lett. 82, 1345 (1999).

[46] C. Zhang, Y.-F. Huang, Z. Wang, B.-H. Liu, C.-F. Li, and G.-C. Guo, Experimental Greenberger-Horne-Zeilinger-Type Six-Photon Quantum Nonlocality, Phys. Rev. Lett. 115, 260402 (2015).
[47] X.-L. Wang, L.-K. Chen, W. Li, H.-L. Huang, C. Liu, C. Chen, Y.-H. Luo, Z.-E. Su, D. Wu, Z.-D. Li et al., Experimental Ten-Photon Entanglement, Phys. Rev. Lett. 117, 210502 (2016).

[48] C. F. Roos, M. Riebe, H. Häffner, W. Hänsel, J. Benhelm, G. P.T. Lancaster, C. Becher, F. Schmidt-Kaler, and R. Blatt, Control and measurement of three-qubit entangled states, Science 304, 1478 (2004)

[49] Y. Ji, J. Bian, X. Chen, J. Li, X. Nie, H. Zhou, and X. Peng, Experimental preparation of Greenberger-Horne-Zeilinger states in an Ising spin model by partially suppressing the nonadiabatic transitions, Phys. Rev. A 99, 032323 (2019).

[50] M. Neeley, R. C. Bialczak, M. Lenander, E. Lucero, M Mariantoni, A. D. O'Connell, D. Sank, H. Wang, M. Weides, J. Wenner et al., Generation of three-qubit entangled states using superconducting phase qubits, Nature (London) 467, 570 (2010).

[51] L. Dicarlo, M. D. Reed, L. Sun, B. R. Johnson, J. M. Chow, J. M. Gambetta, L. Frunzio, S. M. Girvin, M. H. Devoret, R. J. Schoelkopf et al., Preparation and measurement of three-qubit entanglement in a superconducting circuit, Nature (London) 467, 574 (2010).

[52] R. Barends, J. Kelly, A. Megrant, A. Veitia, D. Sank, E. Jeffrey, T. C. White, J. Mutus, A. G. Fowler, B. Campbell et al., Superconducting quantum circuits at the surface code threshold for fault tolerance, Nature (London) 508, 500 (2014).

[53] D. Cruz, R. Fournier, F. Gremion, A. Jeannerot, K. Komagata, T. Tosic, J. Thiesbrummel, C. L. Chan, N. Macris, M.-A. Dupertuis, and C. Javerzac-Galy, Efficient quantum algorithms for GHZ and $W$ states, and implementation on the IBM quantum computer, Adv. Quantum Technol. 2, 1900015 (2019).

[54] M. Treinish and D. M. Rodríguez, GHZ state example, GitHub, https://github.com/Qiskit/qiskit-terra/blob/master/ examples/python/ibmq/ghz.py.

[55] Qiskit, Measurement error mitigation, Qiskit 0.19.6 documentation, https://github.com/Qiskit/qiskit-terra/blob/ master/examples/python/ibmq/ghz.py.

[56] Qiskit, Tensored measurement calibration, Qiskit 0.19.6 Documentation, https://qiskit.org/documentation/stubs/qiskit.ignis. mitigation.tensored_meas_cal.html.

[57] IBM-Q-Team, IBM-Q-5 Yorktown backend specification v2.0.0, 2019.

[58] IBM-Q-Team, IBM-Q-15 Melbourne backend specification v2.0.0, 2019.

[59] IBM-Q-Team, IBM-Q-53 Rochester backend specification v1.2.0, 2020. 